\title{
Harnessing Nutritional Benefits of Spirulina platensis: Standardization of Cultivating Conditions of Spirulina in Kilimanjaro
}

\author{
David O. Nyakundi" and Phocus Cleophas \\ Mwenge Catholic University, P. O. Box 1226, Moshi-Tanzania. \\ *Corresponding author: onchongadavid@hotmail.com; david.nyakundi@mwecau.ac.tz \\ Received 21 Jun 2021, Revised 22 Sep 2021, Accepted 25 Sep 2021, Published Oct 2021 \\ DOI: https://dx.doi.org/10.4314/tjs.v47i4.7
}

\begin{abstract}
Malnutrition remains a challenge in Tanzania, notwithstanding government initiatives and health education geared towards assuaging the problem. According to the World Health Organization (WHO), there will be more than 600,000 severely malnourished children in Tanzania by the year 2030. In particular, protein based malnutrition remains the greatest challenge due to the irreplaceable nature of its essential amino acids. Macronutrients and micronutrients which are found in Spirulina platensis have been recommended by WHO to address malnutrition in developing countries. Spirulina platensis is a filamentous Cyanobacterium microalgae with the highest recorded protein content of plant origin with several immune boosting nutrients. Spirulina cultivation requires sufficient aeration, proper light intensity and salinity for maximum biomass yield, cell productivity, specific growth rate, and protein content. This paper presents the findings of a study carried out in Kilimanjaro on the optimized conditions, locally and economically tailored approach system required to grow spirulina in the region. The study established the use of food grade organic media with low-cost urea as nitrogen source, a greenhouse average temperature of $30-32{ }^{\circ} \mathrm{C}$ in the months between December to March, alkalinity of $5 \mathrm{~g} / \mathrm{L}$, mixing frequency of 3 times per day/100 L and partial shading, as the optimum conditions for outdoor cultivation of spirulina.
\end{abstract}

Keywords: Malnutrition, Spirulina, Protein, growth condition.

\section{Introduction}

Spirulina platensis (now called Athrospira platensis) belongs to a group of planktonic photosynthetic organisms. It is found in the family of blue-green algae and classified as bacterium due to its prokaryotic structure (Koru 2012 Sudhakar et al. 2014). Spirulina is reckoned to be the oldest living plant that emerged almost 3.6 billion years ago and served as an evolutionary link between green plants and bacteria that generated atmospheric oxygen for all subsequent life forms (Soni et al. 2017). Spirulina has higher $\mathrm{CO}_{2}$ fixing efficiency and grows naturally in water bodies with high alkalinity in subtropical climates
(Anupama and Ravindra 2000, Sjors and Alessandro 2010). Similar to higher plants, spirulina contains chlorophyll hence classified as microalgae of the genus Athrospira. The name Spirulina is commonly and commercially used to describe this genus for possessing cellular spiral trichromes. To date, there are four known species of Spirulina, namely; $S$. maxima, $S$. platensis, $S$. Jenneri and $S$. fusiformis (Kaggwa et al. 2013). The two species; S. maxima and S. platensis have gained commercial applications in food and pharmaceutical industries because of their relevance as food supplements (Salunke et al. 2016, Gonçalves et al. 2016). Spirulina was

1412 
discovered by Hernando Cortez and Pierre Dangeard in the year 1519 in Lake Texcoco where they observed it to be a sole sustaining diet for flamingoes. Later, people started using and commercializing it as food. The first spirulina processing plant, Sosa Texcoco, was set up in 1969 by the French (Ugwu et al. 2008).

Studies have revealed that, the bioavailability of nutrients in spirulina is higher than that of dietary fiber (Cruchot 2008 and Falquet and Hurni 2006). Spirulina contains highest ever known plant proteins (60-70\%) with $47 \%$ essential amino acids and essential fatty acids. It also contains beta-carotene and carotenoid thus making it an excellent antioxidant with vitamins and mineral elements (Charpy et al. 2008, Zeinalian et al. 2017, Soni et al. 2017). Moreover, spirulina is particularly rich in vitamin B12 (cobalamin), a vitamin hardly found in any vegetarian diet (Platt and Jassby 1976). It also contains vitamin E and iron akin to one found in wheat gram (Yin et al. 2017). Spirulina also contains minerals such as magnesium, calcium, and phosphorus in amounts comparable to animal sources such as fish and rich in chromium, copper, potassium, sodium and zinc (Cuellar-Bermudez et al. 2015, Zeinalian et al. 2017). It also contains essential polyunsaturated fatty acids with cell wall polysaccharides of $86 \%$ digestibility, making it easier to assimilate in human body (Piorreck et al. 1984, Sjors and Alessandro 2010).

Due to its nutritional benefits, spirulina can be used to address the problem of global acute malnutrition. Indeed, administration of spirulina at a dose of 10 grams per day seemed to significantly and quickly improve the nutritional status of undernourished children in the intervention group when compared to the control group (Matondo et al. 2016). Certainly, the United Nations has avowed spirulina as the best food for the future (Pulz and Gross 2004), with UNESCO declaring it as the most ideal food for tomorrow. NASA and European Space Agencies have also identified spirulina as the main food to be used in long-term space missions and authorized as one of the best protein sources against malnutrition by Food and Drug Association (FDA). The phycocyanin pigment contained in spirulina, can be extracted and used in the cosmetic and food industries. When phycocyanin is highly purified, it has fluorescence properties that are used in immune diagnostic tests (SevrinReyssac 1998).

Spirulina growth requirements are similar to those of common terrestrial plants. However, it relatively uses less water and has high efficiency of resource utilization (Sudhakar et al. 2014). Cultivation of spirulina is advantageous over conventional agriculture due to its high protein biomass, less land requirements, easy harvesting, and suitability for cultivation in arid areas and in saline water (Sopandi et al. 2011, Zeng et al. 2011). Successful large scale outdoor cultivation, growth and production of spirulina, depend on a number of factors (Pandey et al. 2010). Such factors include availability of nutrients, temperature, light intensity, alkalinity, aeration, and secchi depth (Pandey et al. 2010, Rinanti et al. 2013, Huesemann et al. 2016. However, high biomass production of spirulina can be expensive due to the cost of minerals required to make suitable cultivation media where it is usually cultured in Zarrouk's media (Zarrouk 1966).

This paper reports a study that was carried out to assess environmental and meteorological conditions required for successful culturing, cultivation and growth of spirulina in Kilimanjaro. The $S$. platensis was cultivated in natural water from Kilimanjaro springs in the presence of the selected medium majorly made up of urea, sea salt and sodium bicarbonate. Urea was used as a less expensive source of nitrogen as compared to conventional nitrate sources that are typically used in spirulina culturing (Soni et al. 2019). However, the amount of urea used was carefully regulated since levels higher than $300-500 \mathrm{mg} \mathrm{L}^{-1}$ have been found inhibitory and toxic to spirulina (Torre et al. 2003). To obtain high spirulina biomass from outdoor cultivation, shading was 
Nyakundi and Cleophas - Harnessing nutritional benefits of Spirulina platensis ...

found to be necessary to avoid high temperature and light intensities. The effect of light intensity, temperature, agitation/mixing frequency and alkalinity on growth rate and biomass production of spirulina were investigated. Due to vulnerability to contamination, outdoor cultivation of spirulina is often difficult, but in this study, spirulina was grown under a greenhouse to protect it from foreign particles in the air that are often the main sources of contamination. The use of the greenhouse also optimized biomass yield by ensuring controlled growth environment especially fluctuating diurnal temperature.

It is undeniable fact that malnutrition is a threat in the developing world and more specifically in Tanzania. This calls for concerted efforts by the government and nongovernmental organizations to continue working on sustainable measures aimed at addressing the problem of malnutrition with its resultant negative impacts. Indeed, spirulina has been recommended to be used in addressing malnutrition and most West African countries have invested in this wonder microalga with plenty of available evidence on the positive effects of using it against malnutrition and mitigating the blunt of HIV AIDS. However, research on spirulina and its likely impacts, has not received much emphasis in East Africa. This study was therefore carried out to establish the optimum conditions which can guarantee the cultivation and growth of spirulina in Kilimanjaro Tanzania. Furthermore, the study sought to establish the kind of locally tailored, easily adoptable and economically feasible set up system which can be used to cultivate and produce spirulina in Kilimanjaro.

\section{Materials and Methods \\ Location and cultivation conditions}

This study was conducted in Kilimanjaro region in Moshi District Tanzania for the purpose of establishing the optimum environmental growth conditions required for outdoor cultivation of spirulina. The weather conditions in Kilimanjaro vary across the twelve months in the year. The culturing of spirulina was done for seven months (from December 2018 to June 2019) for the purpose of studying the months with the best temperature favorable for spirulina growth. Moreover, Moshi being near Mount Kilimanjaro, the temperature fluctuate highly, especially during the day with the increase of altitude. This study was carried out specifically in Mwenge Catholic University, located approximately 10 kilometers from Moshi Town (Latitude 9634854 N, Longitude 313565E, Elevation $1019 \mathrm{~m})$. The strain of Spirulina platensis used was obtained from Mwenge Catholic University where it was previously maintained in modified Zarrouk's medium at ambient temperature.

\section{Media and mother culture}

Portable water from Kilimanjaro Mountain was used. The used water essentially determined the constitution of the media (fundamentally modified from Zarrouks media). The media were mainly made up of urea, sulphate, chloride, nitrate, sodium, nitrate, phosphate, magnesium and calcium, all supplied as organic fertilizers. Crystallized fertilizer and food grade chemicals were used for cost reduction purposes but solubilized before use. Table 1 shows the formula of the media culture used that was derived and modified from Zarrouks media (Table 2; Zarrouk 1966). Fully grown concentrated spirulina culture was used for inoculum preparation. Spirulina strain with a high proportion of coiled filaments $(<25 \%$ straight filaments, or none) with clearly green color was selected.

\section{Experimental setup}

The media were prepared according to the recipe indicated in (Table 1), which was basically modified from Zarrouk's medium, with urea serving as the main source of nitrogen. All the reagents used $\left(\mathrm{NaHCO}_{3}\right.$, $\mathrm{NaNO}_{3}, \mathrm{MgSO}_{4}$, monophosphate and $\mathrm{NaCl}$ ) for cultivation media were of food grade (commonly used fertilizers for organic farming 
obtained from Balton Company, Arusha). The bicarbonate used in the study was obtained from Moshi common market sold as baking powder and common sea salt was used instead of high grade $\mathrm{NaCl}$. Spirulina was cultivated in the outdoors within a greenhouse in $100 \mathrm{~L}$ common plastic basins. The greenhouse was used purposely to tame rapid fluctuations of temperature, especially in the night hours. Part of the greenhouse was shaded using a black material to reduce light intensities and allow subsequent experimental set up. The factors that could influence optimum spirulina biomass production in Kilimanjaro such as temperature, light intensity, alkalinity and aeration rate (mixing frequency) were determined accordingly. The measurement of turbidity was used as a function of spirulina biomass or cell concentration using Secchi disk (where the disk could be lowered into the culture until was no longer seen, and the depth at which it disappeared was recorded). The cell concentrations were also measured using the optical density (OD) at $450 \mathrm{~nm}$ using spectrophotometer (Thermo Fisher Scientific). The depth of the culture was maintained constant throughout the experiment by addition of water and commensurate nutrients. The amount of culture and thus its depth, could change from time to time (reduce) due to evaporation and consumption of water by the cells. The addition of lost and consumed water would require to be added from time to time to maintain the desired culture depths.

Table 1: Composition of cost effective medium (modified from Zarrouk's medium)

\begin{tabular}{lc}
\hline Composition of modified Zarrouk's medium \\
\hline Fertilizer/Chemical/Salt & Quantity $(\mathrm{g}) / \mathrm{L}$ \\
\hline Bicarbonate & 16 \\
$\mathrm{NaNO}_{3}$ & 1.50 \\
$\mathrm{NaCl}$ & 5.0 \\
$\mathrm{~K}_{2} \mathrm{SO}_{4}$ & 1.0 \\
$\mathrm{Monophosphate}_{\text {Urea }}$ & 0.5 \\
$\mathrm{MgSO}_{4}$ & 0.35 \\
$\mathrm{Iron}$ & 0.2 \\
$\mathrm{CaCl}_{2}$ & 0.01 \\
& 0.04 \\
\hline
\end{tabular}

Table 2: Composition of the Zarrouk's medium

\begin{tabular}{lc}
\hline \multicolumn{2}{c}{ Composition of Zarrouk's medium } \\
\hline Fertilizer/Chemical/Salt & Quantity (g)/ L \\
\hline $\mathrm{NaHCO}_{3}$ & 16.8 \\
$\mathrm{NaNO}_{3}$ & 2.50 \\
$\mathrm{NaCl}$ & 1.0 \\
$\mathrm{~K}_{2} \mathrm{SO}_{4}$ & 1.0 \\
$\mathrm{~K}_{2} \mathrm{HPO}_{4}$ & 0.5 \\
$\mathrm{MgSO}_{4} \cdot 7 \mathrm{H}_{2} \mathrm{O}$ & 0.2 \\
$\mathrm{FeSO}_{4} \cdot 7 \mathrm{H}_{2} \mathrm{O}$ & 0.01 \\
$\mathrm{CaCl}_{2} .2 \mathrm{H}_{2} \mathrm{O}$ & 0.04 \\
EDTA & 0.08 \\
\hline
\end{tabular}

\section{Effects of temperature on growth of $S$. platensis}

The effects of temperature on the growth of Spirulina platensis over a period of seven months were determined. The daily temperature measurements were determined using a digital thermometer (Thomas Scientific). The weekly and monthly averaged temperature measurements were recorded against averaged spirulina biomass measurements. The average temperature was compared to the average growth of spirulina and recorded accordingly from all triplicate samples. The $\mathrm{OD}_{450}$ and Secchi depth of the culture were measured as functions of spirulina biomass. All other growth factors were kept constant, i.e. five minutes stirring of the culture in three times a day, alkalinity $\left(5 \mathrm{~g} / \mathrm{L}^{-1} \mathrm{NaCl}\right.$ : $16 \mathrm{~g} / \mathrm{L}^{-1} \mathrm{NaHCO}_{3}$ determined earlier) in the greenhouse under partial shading and same medium.

\section{Effects of light intensity on growth of $S$. platensis}

To determine the effects of natural light intensity on the growth of $S$. plantensis, three categories of culture samples were established. The first category had a set placed in the greenhouse under partial shading, the second category had a set placed in the greenhouse without shading and the third category had a set placed outside the greenhouse without shading. All sets were in triplicates. Earlier 
Nyakundi and Cleophas - Harnessing nutritional benefits of Spirulina platensis ...

determined alkalinity of $5 \mathrm{~g} / \mathrm{L}^{-1} \mathrm{NaCl}: 16 \mathrm{~g} / \mathrm{L}^{-1}$ $\mathrm{NaHCO}_{3}$ and stirring/mixing frequencies (five minutes in three times a day) were kept constant under the same Zarrouk modified medium. Spirulina biomass from respective samples was determined accordingly over the period of seven weeks.

Effects of alkalinity on growth of $S$. platensis

Alkalinity is the ability of the solution to resist change of $\mathrm{pH}$ which is pertinent to the growth of spirulina. Spirulina being alkaliphilic microalgae, a stable alkalinity is required for not only its growth but also to avoid contaminations by other ubiquitous microorganisms. The effects of $\mathrm{NaCl}$ and $\mathrm{NaHCO}_{3}$ (used in this study as a function of alkalinity) on growth of spirulina culture were determined accordingly. The modified Zarrouk medium was prepared with different levels of $\mathrm{NaCl}(0 \mathrm{~g}, 2 \mathrm{~g}, 5 \mathrm{~g}, 8 \mathrm{~g}, 11 \mathrm{~g}, 14 \mathrm{~g}$ and $17 \mathrm{~g})$ per litre and the effects of the different levels of alkalinity on the growth of Spirulina plantensis were determined from the average growth of culture taken for seven weeks. A sample with zero grams of sodium chloride $(\mathrm{NaCl})$ in Zarrouk modified medium served as a control. The amount of $\mathrm{NaHCO}_{3}\left(16 \mathrm{~g} / \mathrm{L}^{-1}\right)$ was kept constant in all samples. The samples were maintained in triplicates.

\section{Effects of mixing rate/ aeration on growth of S. platensis}

To determine the effects of mixing rate on spirulina growth, three sets of culture of spirulina (in triplicates) were maintained under a greenhouse with partial shading in medium with constant alkalinity of $5 \mathrm{~g} / \mathrm{L}^{-1} \mathrm{NaCl}: 16 \mathrm{~g} / \mathrm{L}$

${ }^{1} \mathrm{NaHCO}_{3}$. The $\mathrm{pH}$ was constantly monitored to ensure alkalinity was maintained at saline levels during the period of study. The entire samples were mixed for the same time (5 minutes) but with different frequencies of four, three and two times in a day. The effects of mixing frequency on the growth of spirulina was determined weekly for seven months by measuring Secchi depth. Mixing was done manually by use of hand brush and regular microscopic observations were carried out to determine the effects of shear pressure introduced by the mixing. Inverted light microscope (Olympus, CKX41, and Tokyo, Japan) was used.

\section{Statistical analysis}

The data obtained from the study was analyzed using excel statistical analysis and represented empirically.

\section{Results and Discussions \\ Effects of temperature on growth of $S$. platensis}

There is ample evidence pointing to the critical role of temperature on spirulina biomass production. Seasonal and diurnal temperature variations represent the greatest bottleneck for spirulina biomass production especially in the tropical regions like Kilimanjaro. Cultivation of spirulina in open pond is often unachievable due to difficulties in maintaining required optimal temperature of 29-35 ${ }^{\circ} \mathrm{C}$ throughout the day and year (Tomaselli et al. 1987). Spirulina being Cyanobacteria with chlorophyll $\mathrm{A}$, will require temperature and water in order to carry out oxygenic photosynthesis; a chemical reaction that will yield glucose for energy (Singh et al. 2016). However, too much heat will denature the enzymes used during the process leading to slowing down of photosynthesis (Singh et al. 2016). In this study; the temperature under which spirulina was cultivated was recorded and averaged monthly. The maximum growth of spirulina was observed during the months of December to March when there were relatively higher seasonal temperature. The monthly growth of spirulina was recorded daily using the OD and the Secchi depth as functions of spirulina biomass increase or decrease (Figure 1a and Figure 1b). The measurement of both the Secchi depth and the OD were the lowest in the month of February that corresponded with an average of $32{ }^{\circ} \mathrm{C}$, indicating the highest spirulina cell concentrations, thus high growth rate. The recorded temperature were for the samples kept and partially shaded under the 
greenhouse settings. The rest of the factors such as mixing frequencies and alkalinity were maintained relatively constant with the $\mathrm{pH}$ of the culture maintained at between 9.5 and 10 . Minimum growth of spirulina was observed when the temperatures were below $25{ }^{\circ} \mathrm{C}$ especially in the months of May and June which corresponded to lowest spirulina biomass production. The results obtained were comparable to ones previously reported where, the culture conditions of $S$. platensis at the temperature of $28-30{ }^{\circ} \mathrm{C}$ and high light intensity of 180-192 $\mu \mathrm{mol}$ photons $\mathrm{m}^{-2} \mathrm{~s}^{-1}$, stimulated greater growth (Lee et al. 2012).

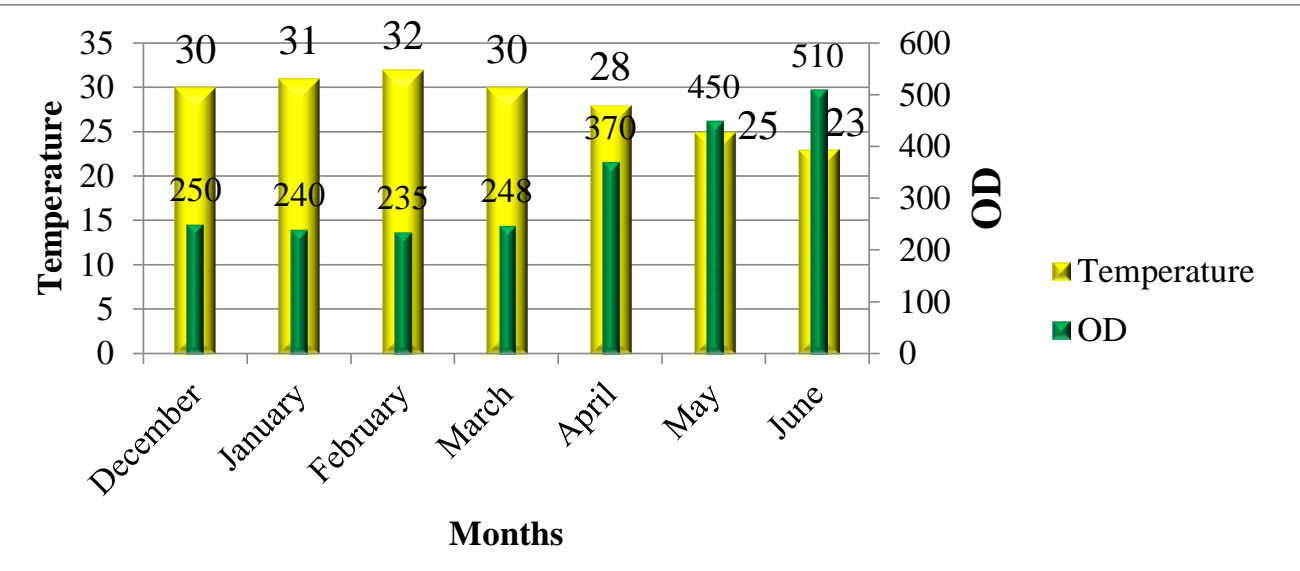

Figure 1(a): The effects of temperature on spirulina growth as determined by measurement of Optic Density (OD) at $450 \mathrm{~nm}$. The growth rate was highest in the months of December to March.

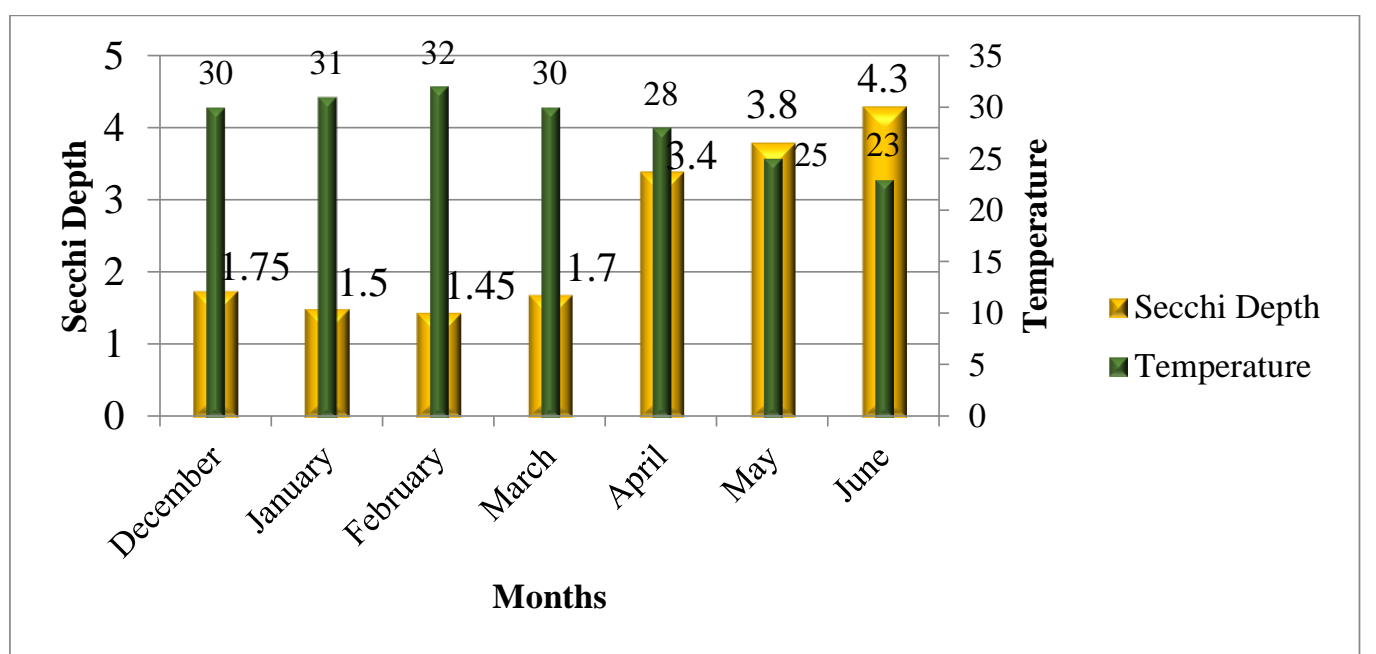

Figure 1(b): The effects of temperature on spirulina growth as determined by measurement of Secchi depth. The growth rate was highest in the months with seasonally high temperature in Kilimanjaro. 
Nyakundi and Cleophas - Harnessing nutritional benefits of Spirulina platensis ...

Effects of light intensity on growth of $S$. platensis

Similar to other photoautotrophic organisms, spirulina converts light energy into chemical energy through photosynthesis, and indeed, optimum growth of spirulina depends on the quality, intensity and duration of light (Novoveská et al. 2016). This is because spirulina makes its own food in the presence of optimal light (sunlight) within some specific range (Sudhakar et al. 2014). High values of light intensity promote spirulina growth and low values result in a biomass that is rich in pigments and proteins (Trabelsi et al. 2009). Maximum cell concentration is achieved with increased light intensity due to accelerated photosynthetic production of ATP and NADPH. However, such increase is dependent on the biomass concentrations. When certain biomass concentration is attained, any increase in light intensity ceases to have any effect on growth most likely due to photo saturation and shadowing. Spirulina will also require dark periods; moments when photosynthetic chemical reactions take place during which synthesis of proteins and respiration occurs. The study established outdoor optimum light requirements that can be easily adopted by the local people for spirulina growth. The triplicate culture samples kept under partial shading under the greenhouse, recorded the highest growth followed by the culture samples that were under the greenhouse (Figure 2). However, it should be noted that the growth of spirulina among the culture samples that were kept outside the greenhouse could as well have been affected by nocturnal temperature, thus recording the highest Secchi for lower concentrations of cells. However, there were observable differences between the culture samples that were shaded under the greenhouse and those that were unshaded under the same conditions. From the findings, it is evident that high light intensity affects the growth of spirulina, most probably due to photo inhibition that reduces cellular growth rate and photoxidation which causes severe cell damage (Vonshak et al. 1994). Indeed, ultraviolet light from the sun can damage the algal DNA hence inhibiting the photosynthetic pathway. On very bright days, UV-B radiation can diminish photosynthesis by $8.2 \%$ (Helbling et al. 1994). This explains why the rates of photosynthesis accelerate during the morning hours, and decelerate at noon time when the radiation levels are highest. It was on the basis of this understanding that the experiment was carried out in this study to establish the light conditions that can guarantee optimum growth of Spirulina platensis in hybrid (use of green house) outdoor conditions. The observations made in this study concur with the previously carried out studies (Trabelsi et al. 2009).

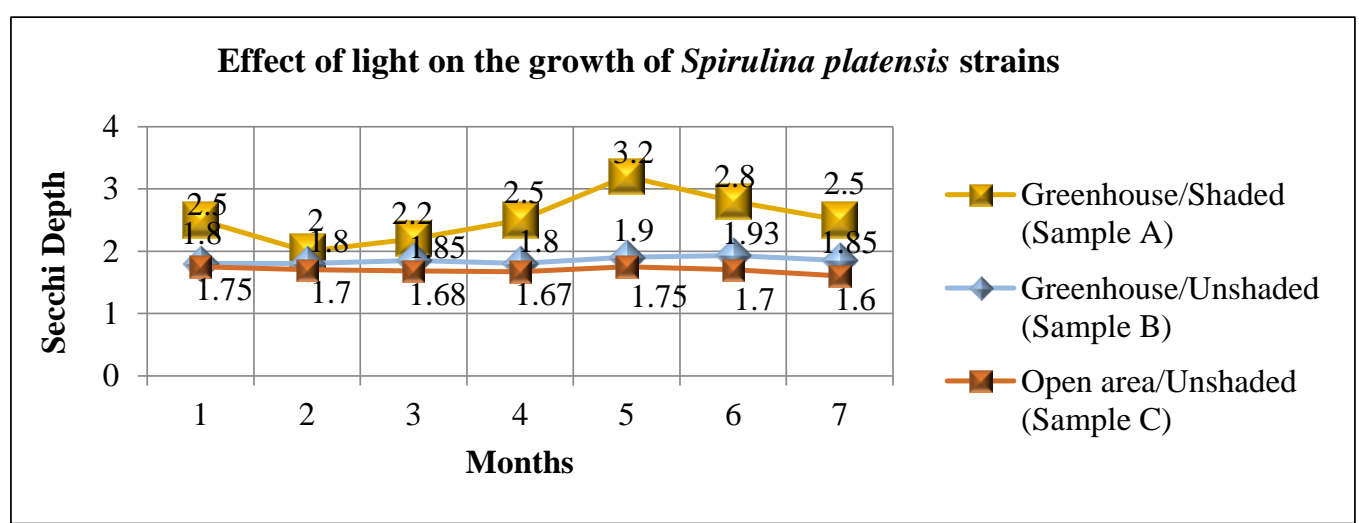

Figure 2: The effects of light intensity on spirulina growth as determined by measurement of Secchi depth. The growth rate was highest in cultures cultivated in the green house under partial shading. 
Effects of alkalinity on growth of S. platensis

High alkalinity favors the growth of spirulina and helps in reducing harmful bacteria and fungi invasions (Jourdan 2006). Alkalinity is the ability of the culture to stabilize and grow within a given suitable $\mathrm{pH}$. In this study, alkalinity of the culture was a function of sodium chloride and sodium bicarbonate salt combinations. In order for the spirulina culture to grow and remain free of contamination, the $\mathrm{pH}$ must be above 7.5 and below 11.5. Beyond a $\mathrm{pH}$ value of 11.5 , the cultivation of $S$. platensis is delayed (Seshadri and Thomas 1978). The amount of sodium chloride to be used can preferentially differ on the basis of the source of water. For instance, the use of sea water may not require any adjustment of salinity, i.e. addition of sodium chloride. This study determined the appropriate amount of sodium chloride that could be added in combination with sodium bicarbonate to provide the best alkalinity that could in return yield optimum spirulina biomass in Kilimanjaro. To the six samples of spirulina culture, different amounts of $\mathrm{NaCl}$ was added in addition to $\mathrm{Na}_{2} \mathrm{CO}_{3}\left(16 \mathrm{~g} / \mathrm{L}^{-1}\right)$. The $\mathrm{pH}$ of samples (as an indicator of stable alkalinity) and Secchi depth (as an indicator of growth) were taken and averaged weekly. The culture sample with no added $\mathrm{NaCl}$ recorded the lowest $\mathrm{pH}$ of 8.1 and highest average Secchi, indicating slow growth of the culture (Figure 3). This study established the use of $5 \mathrm{~g} / \mathrm{L}^{-1}$ $\mathrm{NaCl}$ and $16 \mathrm{~g} / \mathrm{L}^{-1} \mathrm{Na}_{2} \mathrm{CO}_{3}$, and as reported by Soni et al. (2016), and Montagnes and Franklin (2001), to be the best in achieving optimum alkalinity and the stable $\mathrm{pH}$ of 10 that yielded highest spirulina biomass. Correspondingly, use of $\mathrm{NaCl}$ beyond $5 \mathrm{~g} / \mathrm{L}^{-1}$, resulted in higher alkalinity with relatively higher $\mathrm{pH}$ and Secchi depths that recorded lowest biomass. It was obvious from this study that, higher alkalinity slowed down spirulina growth due to decrease of available carbon dioxide for photosynthesis (Singh and Dhar 2019). The use of $\mathrm{NaHCO}_{3}$ was informed by the fact that, only carbonate and bicarbonate ions are available to submerged photosynthetic plants in water with high pH (Poschenrieder et al. 2018).

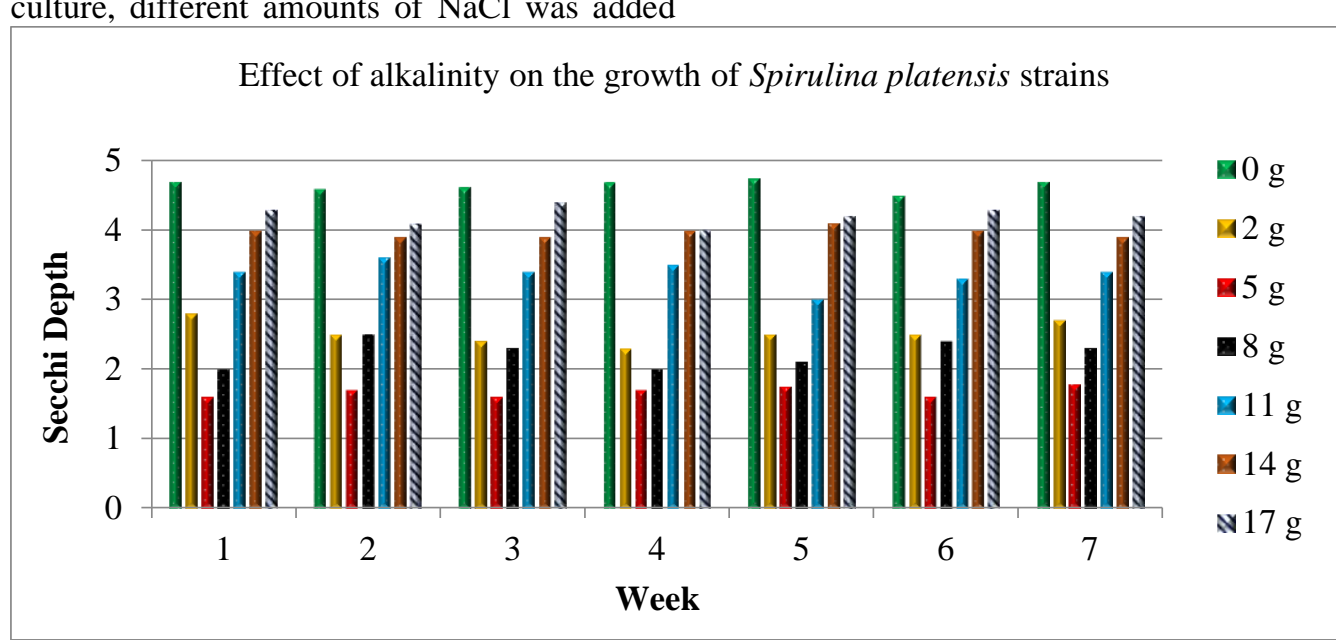

Figure 3: The effects of alkalinity on spirulina growth as determined measurement of Secchi depth.

Effects of mixing frequency on growth of $S$. platensis

The purpose of mixing spirulina culture is to ensure proper aeration. Under commercial production of spirulina, aeration is achieved by rotators to provide agitation and suspension of growing cells (Dubey 2006). In this study, the samples of spirulina culture were mixed in the basins to provide required aeration to the culture. In order to produce ultrahigh density 
Nyakundi and Cleophas - Harnessing nutritional benefits of Spirulina platensis ...

cultures of spirulina, it is necessary to agitate the culture to homogenize it and ensure good distribution of lighting (adequate exposure to illumination) among spirulina filaments. Moreover, mixing ensures good aeration (bubbling with air) which helps to uniformly distribute carbon dioxide concentrations, maintain even distribution of nutrient and remove inhibitory excess oxygen (Dubey 2006). Indeed, continuous mixing of the culture medium is required to prevent cell sinking and thermal stratification. When aeration is not adequate, the efficiency of energy utilization and biomass production will be low since the cell on the surface of the medium will float due to the presence of air-filled vacuoles. The floated cells will be photo-inhibited, resulting in low growth or low biomass production. Additionally, lack of optimum mixing, will result to uneven increased spirulina cell concentrations that will increase self-shading and decrease growth rate. However, aeration should be done at optimized conditions, i.e. optimized velocities since low velocities result in dead zones around corners, while high velocities incur high energy cost, and may result in shear stress that damages the algal cells. Moreover, not all algal species tolerate vigorous mixing and this calls for the need to create the best agitating conditions. This study determined the maximum aerations/ agitation rates (determined in terms of mixing frequency) necessary for outdoor cultivation of spirulina. The mixing of 3 to 4 times a day for five minutes was found to be the best for optimum production of spirulina (Figure 4) in $100 \mathrm{~L}$ basin.

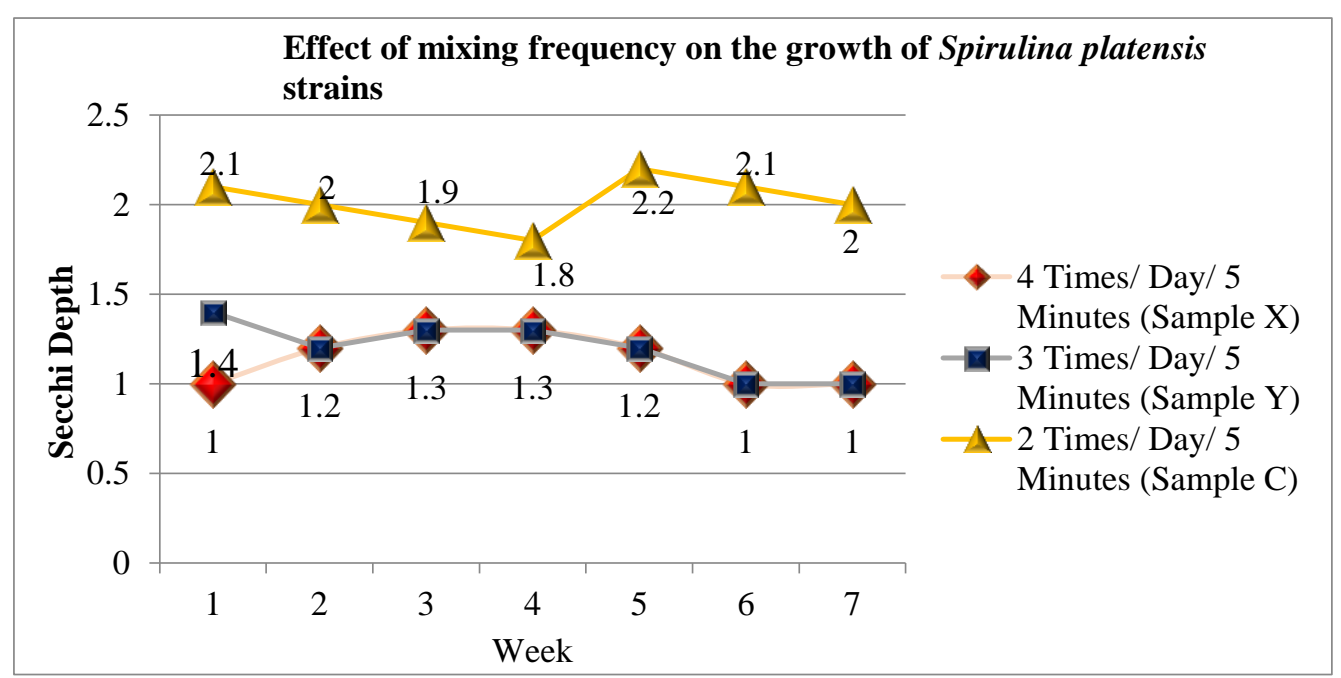

Figure 4: The effects of mixing/ agitation on spirulina growth as determined measurement of Secchi depth.

\section{Conclusion and Future Work}

Spirulina is an alkaliphilic microalga that has been of great interest in commercial applications because of its high nutritional values and blue natural color pigment. Owing to its nutritional values as a protein supplement, it may be essential to use it as a means of fighting malnutrition. Its selective growth conditions such as high $\mathrm{pH}$ and alkalinity, prevent overgrowth of other contaminant organisms even in outdoor openpond cultivations and allow relatively easy quality control. Contrariwise, the utilization of spirulina in solving malnutrition related problems is lagging behind especially in East Africa, notwithstanding available overwhelming evidence pointing to it as a possible solution. This could be partly due to 
lack of available cheaper methods of cultivation and lack of awareness on spirulina's nutritional benefits. Adopting commercially available methods of spirulina cultivation might be a daunting task especially in poverty ravaged regions of Africa. This calls for economically adoptable approaches and hybrid techniques such as greenhouses to ensure optimum hygienically produced spirulina. Additionally, production of spirulina is still confined to specific natural areas while awareness on its immense nutritional values remains scanty. Besides, there is need to come up with optimally standardized culturing conditions that will optimize the production of protein contents in spirulina by considering cheaper and locally tailored alternative growth efficient designs. Spirulina protein concentration is known to range from $65 \%$ to $70 \%$, but maximum protein content reported to date from conventional cultivation systems, is $55 \%$ to $59 \%$. Factors such as light intensity, aeration, $\mathrm{pH}$, alkalinity and temperature affect the growth and nutrient productivity in spirulina growth system. Optimization of such factors will ensure maximum production of spirulina.

In Kilimanjaro region where atmospheric day and night temperature fluctuations are common even during known dry and warm seasons of the year, outdoor spirulina cultivation can be truly challenging. Moreover, rainy seasons alternating with windy and dust air can together increase contamination and encumber any outdoor spirulina cultivation. During dry seasons in Kilimanjaro, sunlight is good but might cause cell photolysis of spirulina in conventional outdoor cultivation practices. To circumvent some of these major meteorological and environmental impediments to successful cultivation of spirulina in Kilimanjaro, we constructed a greenhouse over spirulina cultures and shaded them partially. Increase of spirulina biomass was observed in cultures that were partially shaded during the months of December through February. The use of cheaper farming urea, locally available bicarbonate (mainly used for baking), sea salt, and organic food grade fertilizers constituted culture media where spirulina was cultivated. The water found in Kilimanjaro has mainly neutral alkalinity, it was necessary to establish the best alkalinity that could favor the growth of spirulina in the area. We conclusively state that, it is possible to cultivate spirulina in Kilimanjaro despite the microalgae being environmental specific. The use of greenhouse, shading, mixing of three times a day (in 100 litres plastic basin), alkalinity of $5 \mathrm{~g} / \mathrm{L}^{-1}$ $\mathrm{NaCl} / 16 \mathrm{~g} / \mathrm{L}^{-1}$ and use of food grade fertilizers, can guarantee successful production of spirulina in Kilimanjaro in a good number of months in the year. Future work will involve the estimation of protein and other nutritional components of spirulina grown in Kilimanjaro under the established conditions.

\section{Acknowledgement}

We thank the Mwenge Catholic University for providing enabling environment that facilitated the carrying out of the study.

\section{Competing Interest}

The authors of this manuscript declare that they have no competing interests.

\section{References}

Anupama RP and Ravindra P 2000 Valueadded food: single cell protein. Biotechnol. Adv. 18(6): 459-479.

Charpy L, Langlade MJ, Alliod R 2008 Spirulina can be an Asset to the Health and Development in Africa? Research Institute for Development (IRD), Marseilles, $43 \mathrm{pp}$.

Cruchot H 2008 Spirulina, results and prospects. MSc thesis, University of Franche Comte, Besancon, France.

Cuellar-Bermudez SP, Garcia-Perez J, Rittman BE. and Parra-Saldivar R 2015 Photosynthetic bioenergy utilizing $\mathrm{CO} 2$ : an approach on flue gases utilization for third generation biofuels. J. Clean. Prod. 98: 5365.

Dubey RC 2006 A textbook of Biotechnology. Fourth revised and enlarged edition, $\mathrm{S}$. Hand and Company Limited, pp. 419-421. 
Nyakundi and Cleophas - Harnessing nutritional benefits of Spirulina platensis ...

Falquet J and Hurni JP 2006 Spirulina, Nutritional Aspects. Antenna Technologies, Geneva, Switzerland, 26 pp.

Gonçalves AL, Rodrigues, CM, Pires JCM, Simões, M 2016 The effect of increasing $\mathrm{CO} 2$ concentrations on its capture, biomass production and wastewater bioremediation by microalgae and cyanobacteria. Algal Res. 14: 127-136.

Helbling EW, Villafañ EV, Holm-Hansen O 1994 Effects of ultraviolet radiation on Antarctic marine phytoplankton photosynthesis with particular attention to the influence of mixing. Ultraviolet Radiat. Antarctica: Measurements Biol. Effects 62: 207-227.

Huesemann M, Crowe B, Waller P, Chavis A, Hobbs S, Edmundson S. and Wigmosta $M$ 2016 A validated model to predict microalgae growth in outdoor pond cultures subjected to fluctuating light intensities and water temperatures. Algal Res. 13: 195-206.

Jourdan JP 2006 Cultivate Your Spirulina. Antenna Technologies, Geneva, Switzerland, Pages: 146.

Kaggwa NM, Burian A, Oduor SO, Schaerl M 2013 Ecomorphological variability of Arthrospira fusiformis (Cyanoprokaryota)in African soda lakes. MicrobiologyOpen 2(5): 881-891.

Koru E 2012 Earth Food Spirulina (Arthrospira): Production and Quality Standards. In: El-Samragy Y (Ed) Food Additive, IntechOpen: London, UK, ISBN 978-953-51-0067-6.

Lee MC, Chen YC, and Peng TC 2012 Twostage culture method for optimized polysaccharide production in Spirulina platensis. J. Sci. Food Agric. 92(7): 15621569.

Matondo FK, Takaisi K, Nkuadiolandu AB, KazadiLukusa A and Aloni MN 2016 Spirulina supplements improved the nutritional status of undernourished children quickly and significantly: experience from Kisantu, the Democratic Republic of the Congo. Int. J. Pediatrics 2016.
Montagnes DJS and Franklin M 2001 Effect of temperature on diatom volume, growth rate, and carbon and nitrogen content: reconsidering some paradigms. Limnol. Oceanogr. 46: 2008-2018.

Novoveská L, Zapata AK, Zabolotney JB, Atwood MC and Sundstrom ER 2016 Optimizing microalgae cultivation and wastewater treatment in large-scale offshore photobioreactors. Algal Res. 18: 86-94.

Piorreck M, Baasch LH, Pohl P 1984 Biomass production, total protein, chlorophylls, lipids and fatty acids of freshwater green and blue-green algae under different nitrogen regimes. Phytochem. 23: 207-216.

Platt T and Jassby AD 1976 The relationship between photosynthesis and light for natural assemblages of coastal marine phytoplankton. J. Phycol. 12: 421-430.

Pulz MO and Gross W 2004Valuable products from biotechnology of microalgae. Appl. Microbiol. Biotechnol. 6: 635-648.

Poschenrieder C, Fernández JA, Rubio L, Pérez L, Terés J and Barceló J 2018 Transport and use of bicarbonate in plants: current knowledge and challenges ahead. Int. J. Mol. Sci. 19(5): 1352.

Pandey JP, Tiwari A and Mishra RM 2010 Evaluation of biomass production of spirulina maxima on different reported media. J. Algal Biomass Util. 1: 70-81.

Rinanti A, Kardena E, Astuti DI and Dewi K 2013 Growth response and chlorophyll content of Scenedesmus obliquus cultivated in different artificial media. Asian $J$. Environ. Biol. 1: 1-9.

Salunke KJ, Magar SA, Joshi RR, Wadikar MS 2016 Comparative study on the growth of Spirulina platensis on different culture media. Biosci. Discov. 7(1): 90-92.

Seshadri CV and Thomas S 1978 National solar energy conservation. Bhavnagar India, 7: 41-46.

Sevrin-Reyssac J 1998 Biotreatment of swine manure by production of aquatic valuable biomasses. Agric. Ecosyst. Environ. 68: 177-186. 
Singh J and Dhar DW 2019 Overview of carbon capture technology: Microalgal biorefinery concept and state-of-the-art. Front. Mar. Sci. 6: 1-9.

Singh JS, Kumar A, Rai AN and Singh DP 2016 Cyanobacteria: A precious bioresource in agriculture, ecosystem, and environmental sustainability. Frontiers in Microbiology 7: 529.

Sjors VI and Alessandro F 2010 Algae based biofuels, Applications and coproducts.Environment and natural resources management working paper. Environment climate change. Bioenergy monitoring and assessment.

Soni RA, Sudhakar K and Rana R 2016 Biophotovoltaics and biohydrogenthrough artificial photosynthesis: an overview. Int J. Environ. Sustain. Dev. 15 (3): 313-325.

Soni RA, Sudhakar K, Rana RS 2017 Spirulina-from growth to a nutritional product: A review. Trends in Food Science and Technology 69: 157-171.

Soni RA, Sudhakar K and Rana RS 2019 Comparative study on the growth performance of Spirulina platensis on modifying culture media. Energy Reports 5: 327-336.

Sopandi T, Rohmah S, and Agustina SAT 2020 Biomass and nutrient composition of Spirulina platensis grown in goat manure media. Asian J. Agric. Biol. 8(2): 158-167.

Sudhakar K, Premalatha M and Rajesh M 2014 Large-scale open pond algae biomass yield analysis in India: a case study. Int. J. Sustain. Energy 33: 304-315.

Tomaselli L, Torzillo G, Giovannetti L, Pushparaj B, Bocci F, Tredici M, Papuzzo T, Balloni W. and Materassi R 1987 Recent research on Spirulina in Italy. Hydrobiol. 151/152: 79-82.

Torre P, Sassano CEN, Sato S, Converti A, Gioielli LA and Carvalho JC 2003 Fed- batch addition of urea for Spirulina platensis cultivation-thermodynamics and material and energy balances. Enzyme Microb. Technol. 33: 698-707.

Trabelsi L, Ouada HB, Bacha H and Ghoul M 2009 Combined effect of temperature and light intensity on growth and extracellular polymeric substance production by the cyanobacterium Arthrospira platensis. J. Appl. Phycol. 21: 405-412.

Ugwu CU, Aoyagi $\mathrm{H}$ and Uchiyama H 2008 Photobiorectors for mass cultivation of algae. Bioresource Technol. 99: 4021-4028. Vonshak A, Torzillo G, Tomaseli L 1994 Use of chlorophyll fluorescence to estimate the effect of photoinhibition in outdoor cultures of Spirulina platensis. J. Appl. Phycol. 6: 31-34.

Yin C, Daoust K, Young A, Tebbs EJ and Harper DM 2017 Tackling community undernutrition at Lake Bogoria, Kenya: the potential of Spirulina (Arthrospira fusiformis) as a food supplement. Afric. J. Food Agric. Nutr. Dev. 17(1): 1160311615.

Zarrouk C 1966 Contribution to the study of Cyanophyceae. Influence of various' physical and chemical factors on growth and photosynthesis of Spirulina maxima. $\mathrm{PhD}$ Thesis, University of Paris, Paris.

Zeinalian R, Farhangi MA, Shariat A and Saghafi-Asl M 2017 The effects of Spirulina platensis on anthropometric indices, appetite, lipid profile and serum vascular endothelial growth factor (VEGF) in obese individuals: a randomized double blinded placebo controlled trial. $B M C$ Complement. Altern. Med. 17(1): 225.

Zeng XH, Danquah MK, Chen XD and Lu YH 2011 Microalgae bioengi-neering: Autotrophic cultivation from $\mathrm{CO}_{2}$ fixation to biofuel production. Renew. Sustain. Energy Rev. 15(6): 3252-3260. 